\title{
Half-time-based reflectivity tomography and its application to thermoacoustic tomography
}

Mark A. Anastasio, Xiaochuan Pan, Jin Zhang, Yu Zou, Minghua Xu, et al.

Mark A. Anastasio, Xiaochuan Pan, Jin Zhang, Yu Zou, Minghua Xu, Lihong V. Wang, "Half-time-based reflectivity tomography and its application to thermoacoustic tomography," Proc. SPIE 5373, Medical Imaging 2004: Ultrasonic Imaging and Signal Processing, (28 April 2004); doi: $10.1117 / 12.536710$

SPIE. Event: Medical Imaging 2004, 2004, San Diego, California, United States 


\title{
Half-Time-Based Reflectivity Tomography and its Application to Thermoacoustic Tomography
}

\author{
Mark A. Anastasio ${ }^{a}$, Xiaochuan $\mathrm{Pan}^{b}$, Jin Zhang ${ }^{a}$, Yu Zou ${ }^{b}$, Minghua $\mathrm{Xu}^{c}$ and Lihong Wang ${ }^{c}$ \\ ${ }^{a}$ Department of Biomedical Engineering, Illinois Institute of Technology, Chicago, IL 60616 \\ ${ }^{b}$ Department of Radiology, The University of Chicago, Chicago, IL 60637 \\ ${ }^{c}$ Department of Biomedical Engineering, Texas A\&M University, College Station, TX 77843
}

\begin{abstract}
Thermoacoustic tomography (TAT) is an emerging imaging technique with great potential for a wide range of biomedical imaging applications. In this work, we propose and investigate reconstruction approaches for TAT that are based on the half-time reflectivity tomography paradigm. We demonstrate that half-time reconstruction approaches can produce images in TAT that possess better statistical properties than images produced by use of conventional reconstruction approaches.
\end{abstract}

Keywords: Reflectivity tomography, thermoacoustic tomography, image reconstruction

\section{INTRODUCTION}

Reflectivity tomography is an ultrasonic imaging technique that has been investigated extensively and applied to several biomedical ${ }^{1-3}$ and non-destructive testing ${ }^{4}$ imaging tasks. In reflectivity tomography, an ultrasonic pulse is emitted from a point source into a weakly reflecting object. When the ultrasonic pulse interacts with the object, reflected echos are produced that are measured at the source location as a function of time. Under certain physical assumptions, ${ }^{5,6}$ the measured echo data can be related to two-dimensional (2D) integrals of the object's reflectivity function over a family of spherical surfaces that are centered at the source-receiver point. Consequently, the reconstruction problem in reflectivity tomography is equivalent to that of inverting a spherical Radon transform, or a circular Radon transform in the 2D problem.

Exact and explicit inversion formulae have been derived for 2D and three-dimensional (3D) reflectivity tomography assuming circular ${ }^{5}$ or spherical ${ }^{6,7}$ scanning apertures, respectively. A common feature of these inversion formulae is that require complete knowledge of the reflectivity tomography data function. This indicates that temporally-untruncated measurement data must be acquired at all source-receiver locations on the circular or spherical scanning apertures. We have investigated recently the reduced-scan reconstruction problem in reflectivity tomography, where measurements are only acquired over certain regions of the scanning aperture. ${ }^{8,9}$ Using a potato-peeler procedure, it was demonstrated that the reflectivity function was determined uniquely from untruncated measurements that correspond to source-receiver locations that span at least half of the circular or spherical scanning apertures. We have also investigated the so-called half-time reconstruction problem of reflectivity tomography. Using the previously proposed potato-peeler procedure ${ }^{8}$ and concepts from microlocal analysis, we demonstrated ${ }^{10,11}$ that an image could be reconstructed uniquely and stably from measurement data, acquired at all source-receiver locations on the scanning aperture, that were truncated at delay time $T_{\text {trun }} \geq T_{\text {half }}$. Here, $T_{\text {half }}$ represents twice the time it takes for the probing wavefield to propagate from the source to the center of the scanning aperture. In the language of tomography, at each view angle (i.e., source-receiver position), half-time image reconstruction requires knowledge of the set of integrals over spherical surfaces that have radii less than or equal to the radius of the scanning aperture. In this sense, the half-time reconstruction problem of reflectivity tomography is analogous to the half-detector problem ${ }^{12}$ of X-ray tomography with a parallel-beam tomography.

Thermoacoustic tomography (TAT) is an emerging imaging technique with great potential for a wide range of biomedical imaging applications. ${ }^{13}$ In TAT, a short electromagnetic pulse (e.g., microwave or laser) is used to irradiate a biological tissue. When the electromagnetic pulse is absorbed by the tissue, a thermoacoustic effect

Further author information: Send correspondence to Mark A. Anastasio, Ph.D.: E-mail: anastasio@iit.edu 
results in the emission of acoustic signals that can be measured by use of a wide-band ultrasonic transducer. The objective of TAT is to produce an image that represents a map of the spatially variant electromagnetic absorption properties of the tissue. Such an image may be of great value because a strong correlation exists between electromagnetic absorption in biological tissue and the pathological condition of the tissue. ${ }^{14,15}$ Numerous exact ${ }^{13}$ and approximate ${ }^{16,17}$ reconstruction algorithms have been developed for TAT employing spherical scanning apertures. Equivalently, exact image reconstruction can be accomplished through transformation of the TAT reconstruction problem into the reflectivity tomography reconstruction problem, followed by application of an exact reflectivity tomography reconstruction algorithm. Therefore, TAT can benefit greatly from advancements in reflectivity tomography reconstruction theory.

In this work, we propose and investigate reconstruction approaches for TAT that are based on the halftime reflectivity tomography paradigm. We demonstrate that half-time reconstruction approaches can produce images in TAT that possess better statistical properties than images produced by use of full-time reconstruction approaches. We corroborate our theoretical results using simulated and experimental TAT measurement data.

\section{BACKGROUND}

Below we review the salient aspects of reflectivity tomography and describe how it is related to the imaging model of TAT.

\subsection{Review of reflectivity tomography}

\section{Imaging model and data function}

Consider an acoustic medium that contains a compactly supported region $\mathcal{R}$, residing completely inside a sphere of radius $R_{f}$ centered at the origin. The region $\mathcal{R}$ is characterized by an inhomogeneous compressibility $\kappa(\vec{r})$ and density $\rho(\vec{r})$. Outside of $\mathcal{R}$, the background medium has a homogeneous compressibility $\kappa_{0}$ and density $\rho_{0}$ and is assumed to be lossless. This implies a constant speed of sound in the background medium that is denoted by $c_{0}$. The reflectivity function of the medium, which is assumed to be a bounded and continuous function on $\mathcal{R}$, is defined as

$$
f(\vec{r})=\gamma_{k}(\vec{r})-\gamma_{p}(\vec{r})
$$

where

$$
\gamma_{k}(\vec{r})=\left\{\begin{array}{lll}
\frac{\kappa-\kappa_{0}}{\kappa_{0}} & : & \vec{r} \in \mathcal{R} \\
0 & : & \vec{r} \notin \mathcal{R}
\end{array}\right.
$$

and

$$
\gamma_{p}(\vec{r})=\left\{\begin{array}{lll}
\frac{\rho-\rho_{0}}{\rho_{0}} & : & \vec{r} \in \mathcal{R} \\
0 & : & \vec{r} \notin \mathcal{R}
\end{array}\right.
$$

A spherical measurement aperture with radius $R_{0}>R_{f}$ is centered at the origin and encloses the region $\mathcal{R}$. Let $\vec{r}_{0} \in \Omega_{0}$ where $\Omega_{0}$ denotes the set of vectors in $\mathbb{R}^{3}$ that reside on the surface of the measurement sphere. At time $t=0$, an omni-directional acoustic point source located at position $\vec{r}_{0}$ on the measurement aperture emits a spherical pulse into the region $\mathcal{R}$. As the spherically-diverging pulse propagates into the inhomogeneous region $\mathcal{R}$, echos will be produced that propagate back to the source location and are recorded as a function of time. From knowledge of this echo signal, a data function $g\left(\vec{r}_{0} ; \bar{t}\right)$ can be defined, ${ }^{6}$ where $\bar{t}=c_{0} t$, that is related to the reflectivity function $f(\vec{r})$ as

$$
g\left(\vec{r}_{0} ; \bar{t}\right)=\int_{\mathcal{R}} d^{3} \vec{r} f(\vec{r}) \delta\left(\bar{t}-\left|\vec{r}-\vec{r}_{0}\right|\right)
$$

This indicates that the data function $g\left(\vec{r}_{0} ; \bar{t}\right)$ is equal to integrals of $f(\vec{r})$ calculated over concentric spherical surfaces with radii $\bar{t}$ that are centered at the source-receiver location $\vec{r}_{0}$. The goal of 3D reflectivity tomography is to utilize knowledge of $g\left(\vec{r}_{0} ; \bar{t}\right)$ for $\vec{r}_{0} \in \Omega_{0}$ and $\bar{t} \in\left[0, R_{0}+R_{f}\right]$ (i.e., temporally untruncated data acquired at all source-receiver locations) to determine $f(\vec{r})$ by inverting the spherical Radon transform given in Eqn. (4). Exact inversion formulae have been derived ${ }^{6,7}$ for accomplishing this. 


\section{Half-time reflectivity tomography}

Consider half-time data functions $g_{h t}^{-}\left(\vec{r}_{0} ; \bar{t}\right)$ and $g_{h t}^{+}\left(\vec{r}_{0} ; \bar{t}\right)$ that are defined $\forall \vec{r}_{0} \in \Omega_{0}$ as

$$
g_{h t}^{-}\left(\vec{r}_{0} ; \bar{t}\right)=\left\{\begin{array}{lll}
g\left(\vec{r}_{0} ; \bar{t}\right) & : & 0 \leq \bar{t} \leq R_{0} \\
0 & : & \text { otherwise }
\end{array}\right.
$$

and

$$
g_{h t}^{+}\left(\vec{r}_{0} ; \bar{t}\right)=\left\{\begin{array}{lll}
g\left(\vec{r}_{0} ; \bar{t}\right) & : & R_{0}<\bar{t} \leq R_{0}+R_{f} \\
0 & : & \text { otherwise. }
\end{array}\right.
$$

Existing reconstruction algorithms ${ }^{5}$ assume that the data are complete (un-truncated), and therefore will generally produce distorted images when applied directly to $g_{h t}^{-}\left(\vec{r}_{0} ; \bar{t}\right)$ and $g_{h t}^{+}\left(\vec{r}_{0} ; \bar{t}\right)$. Previously, we have employed a layer-stripping procedure, ${ }^{9-11}$ to explicitly identify redundant information in the (untruncated) data function $g\left(\vec{r}_{0} ; \bar{t}\right)$ and demonstrated that $f(\vec{r})$ is determined uniquely from knowledge of the half-time data functions $g_{h t}^{-}\left(\vec{r}_{0} ; \bar{t}\right)$ or $g_{h t}^{+}\left(\vec{r}_{0} ; \bar{t}\right)$. A similar result has been derived recently by Finch et al ${ }^{7}$ using a different mathematical approach. To date, exact and explicit inversion formulae for reconstruction of $f(\vec{r})$ from knowledge of the half-time data functions $g_{h t}^{-}\left(\vec{r}_{0} ; \bar{t}\right)$ or $g_{h t}^{+}\left(\vec{r}_{0} ; \bar{t}\right)$ remain undiscovered. However, we have demonstrated that accurate images can be reconstructed stably from knowledge of the half-time data functions by use of iterative reconstruction procedures. ${ }^{10,11}$

\subsection{Review of TAT imaging model}

Consider a microwave pulse with time dependence $\delta(t)$ that is used to irradiate a tissue sample. When the microwave pulse is absorbed by the tissue, a thermoacoustic effect results in the generation of a pressure wavefield $p(\vec{r}, t)$ that can be measured by use of a wide-band ultrasonic transducer that is located on the surface of a spherical measurement aperture $\Omega_{0}$ that encloses the sample. Assuming that the tissue has homogeneous acoustic properties (but spatially varying microwave absorption properties), the pressure wavefield $p\left(\vec{r}_{0}, t\right)$ measured as a function of time at location $\vec{r}_{0} \in \Omega_{0}$ can be expresses as ${ }^{13}$

$$
p\left(\vec{r}_{0}, t_{0}\right)=\eta \int d \vec{r} A(\vec{r}) \frac{d}{d t_{0}} \frac{\delta\left(t_{0}-\frac{\left|\vec{r}_{0}-\vec{r}\right|}{c}\right)}{4 \pi\left|\vec{r}_{0}-\vec{r}\right|},
$$

where $A(\vec{r})$ denotes the spatial energy absorption function, $c$ is the velocity of sound in the homogeneous background medium, and $\eta$ is a known constant. The reconstruction problem of TAT is to invert Eqn. (7) for determination of $A(\vec{r})$ from knowledge of $p\left(\vec{r}_{0}, t_{0}\right)$ for $\vec{r}_{0} \in \Omega_{0}$ and $t_{0} \in[0, \infty)$. An exact inversion formula has been derived ${ }^{13}$ that can accomplish this.

A mathematical relationship between the TAT and reflectivity tomography data functions can be derived readily. Specifically, by acting $\int_{0}^{t} d t_{0}$ on both sides of Eqn. (7), one can show that

$$
g_{A}\left(\vec{r}_{0}, \bar{t}=c t\right) \equiv \frac{4 \pi}{\eta} t \int_{0}^{t} d t_{0} p\left(\vec{r}_{0}, t_{0}\right)=\int d^{3} \vec{r} A(\vec{r}) \delta\left(\bar{t}-\left|\vec{r}_{0}-\vec{r}\right|\right)
$$

where $g_{A}\left(\vec{r}_{0}, \bar{t}=c t\right)$ corresponds to the spherical Radon transform of $A(\vec{r})$. Consequently, image reconstruction in TAT can be accomplished by inverting Eqn. (8) via. a reflectivity tomography reconstruction algorithm.

\section{STATISTICAL MOTIVATION FOR HALF-TIME RECONSTRUCTION IN TAT}

By use of Eqn. (8), the half-time reflectivity tomography data functions $g_{h t}^{-}\left(\vec{r}_{0} ; \bar{t}\right)$ or $g_{h t}^{+}\left(\vec{r}_{0} ; \bar{t}\right)$ can be defined from the TAT data function $p\left(\vec{r}_{0}, t_{0}\right)$. To motivate the use of half-time reconstruction approaches in TAT, we will investigate the statistical properties of the transformation given in Eqn. (8). We will treat $\boldsymbol{g}_{\boldsymbol{A}}\left(\vec{r}_{0}, \vec{t}\right)$ and $\boldsymbol{p}\left(\vec{r}_{0}, t_{0}\right)$ as stochastic quantities. Here and elsewhere, boldface and normal typefaces will denote a random variable and its mean, respectively. Consider the variance of $\boldsymbol{g}_{\boldsymbol{A}}\left(\vec{r}_{0}, \bar{t}\right)$ that can be expressed as

$$
\operatorname{Var}\left\{g_{A}\left(\vec{r}_{0}, \bar{t}=c t\right)\right\}=\left(\frac{4 \pi}{\eta} t\right)^{2} \operatorname{Var}\left\{\int_{0}^{t} d t_{0} p\left(\vec{r}_{0}, t_{0}\right)\right\}
$$


or equivalently,

$$
\operatorname{Var}\left\{\boldsymbol{g}_{\boldsymbol{A}}\left(\vec{r}_{0}, \bar{t}=c t\right)\right\}=\left(\frac{4 \pi}{\eta} t\right)^{2} \int_{0}^{t} d t^{\prime} \int_{0}^{t} d t^{\prime \prime} \operatorname{Covar}\left\{\boldsymbol{p}\left(\vec{r}_{0}, t^{\prime}\right), \boldsymbol{p}\left(\vec{r}_{0}, t^{\prime \prime}\right)\right\}
$$

where Covar $\left\{\boldsymbol{p}\left(\vec{r}_{0}, t^{\prime}\right), \boldsymbol{p}\left(\vec{r}_{0}, t^{\prime \prime}\right)\right\}$ denotes the covariance of $\boldsymbol{p}\left(\vec{r}_{0}, t^{\prime}\right)$ and $\boldsymbol{p}\left(\vec{r}_{0}, t^{\prime \prime}\right)$. Equation (9) describes how the second order statistics of the TAT data function determine the variance of the reflectivity tomography data function.

To proceed further, we will assume a stationary and uncorrelated noise model for the TAT data function that is described by

$$
\operatorname{Covar}\left\{\boldsymbol{p}\left(\vec{r}_{0}, t^{\prime}\right), \boldsymbol{p}\left(\vec{r}_{0}, t^{\prime \prime}\right)\right\}=\sigma_{p}^{2}\left(t^{\prime}\right) \delta\left(t^{\prime}-t^{\prime \prime}\right) \delta\left(\vec{r}_{0}^{\prime}-\vec{r}_{0}^{\prime \prime}\right),
$$

where $\sigma_{p}^{2}\left(t^{\prime}\right)>0$ is the variance of $\boldsymbol{p}\left(\vec{r}_{0}, t\right)$. Such a stochastic model could account for randomness in the pressure wavefield or noises introduced during the act of measurement. Substitution from Eqn. (10) into Eqn. (9) results in

$$
\operatorname{Var}\left\{\boldsymbol{g}_{\boldsymbol{A}}\left(\vec{r}_{0}, \bar{t}\right)\right\}=\left(\frac{4 \pi \bar{t}}{\eta c}\right)^{2} \int_{0}^{\frac{\bar{t}}{c}} d t^{\prime} \sigma_{p}^{2}\left(t^{\prime}\right)
$$

Because $\sigma_{p}^{2}\left(t^{\prime}\right)>0$, Eqn. (11) indicates that $\operatorname{Var}\left\{\boldsymbol{g}\left(\vec{r}_{0}, \bar{t}\right)\right\}$ is a monotonically increasing function of $\bar{t}$. Therefore the spherical Radon transform data $\boldsymbol{g}_{\boldsymbol{A}}\left(\vec{r}_{0}, \bar{t}\right)$ that correspond to large $\bar{t}$ may be significantly corrupted by noise. This observation suggests that a half-time reconstruction approach that utilizes the data function $g_{h t}^{-}\left(\vec{r}_{0} ; \bar{t}\right)$ may be statistically favorable because an estimate of $A(\vec{r})$ can be obtained without use of the measurement data that are corrupted most by noise.

In the special case of stationary noise with $\sigma_{p}^{2}\left(t^{\prime}\right)=\sigma_{p}^{2}$, Eqn. (11) reduces to

$$
\operatorname{Var}\left\{\boldsymbol{g}_{\boldsymbol{A}}\left(\vec{r}_{0}, \bar{t}\right)\right\}=\left(\frac{4 \pi}{\eta}\right)^{2}\left(\frac{\bar{t}}{c}\right)^{3} \sigma_{p}^{2}
$$

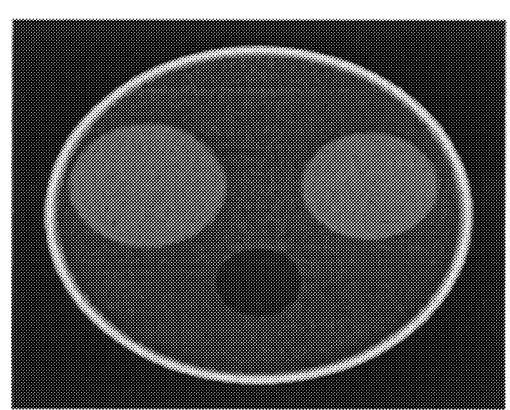

(a)

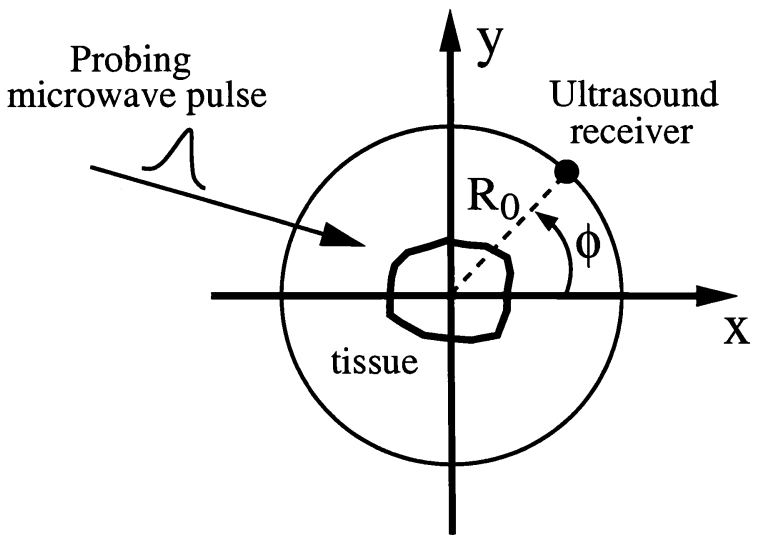

(b)

Figure 1. (a) The mathematical phantom used in the simulation studies. (b) The 2D TAT measurement configuration.

\section{NUMERICAL RESULTS}

We performed numerical studies using simulation and experimental measurement data to investigate the halftime reconstruction approach for $2 \mathrm{D}$ TAT. 


\subsection{Numerical studies}

\section{Simulated TAT measurement data}

The 2D mathematical phantom shown in Fig. 1-(a) was chosen to represent $A(\vec{r})$. From knowledge of $A(\vec{r})$, which had a radius of $R_{f}=1$, the (full-time) data function $g_{A}(\phi, \bar{t})$ was calculated analytically by use of Eqn. (8) assuming the 2D measurement geometry shown in Fig. 1-(b) with $R_{0}=1.01$. Here, the angle $\phi$ replaces $\vec{r}_{0}$ as the indicator of the ultrasound receiver position on the scanning aperture. The data function $g_{A}(\phi, \bar{t})$ was determined at 360 equally spaced positions on the scanning aperture and at 256 equally spaced values of $\bar{t}$ over the interval $\left[R_{0}+R_{f}, R_{0}+R_{f}\right]$. A half-time data function $g_{A}^{h t}(\phi, \bar{t})$ was defined as

$$
g_{A}^{h t}(\phi ; \bar{t})=\left\{\begin{array}{lll}
g_{A}(\phi ; \bar{t}) & : & 0 \leq \bar{t} \leq R_{0} \\
0 & : & \text { otherwise. }
\end{array}\right.
$$

To generate noisy data functions, $\boldsymbol{g}_{A}^{\boldsymbol{h} t}(\phi ; \bar{t})$ and $\boldsymbol{g}_{\boldsymbol{A}}(\phi ; \bar{t})$ were treated as realizations of uncorrelated Gaussian stochastic processes with means of $g_{A}^{h t}(\phi ; \bar{t})$ and $g_{A}(\phi ; \bar{t})$, respectively. Motivated by Eqn. (12), the variances of the distributions were determined according to the rule

$$
\sigma^{2}=0.025 \bar{t}^{3} \text {. }
$$

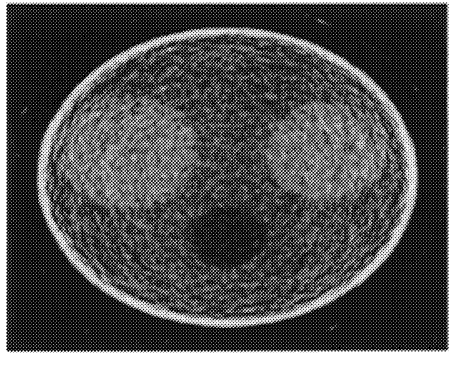

(a)

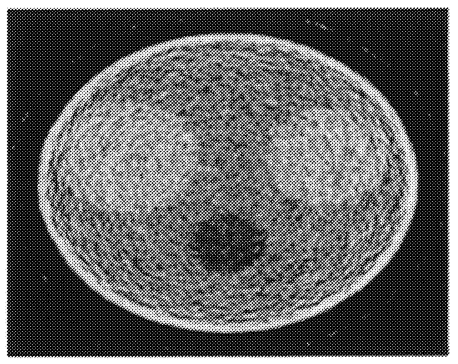

(c)

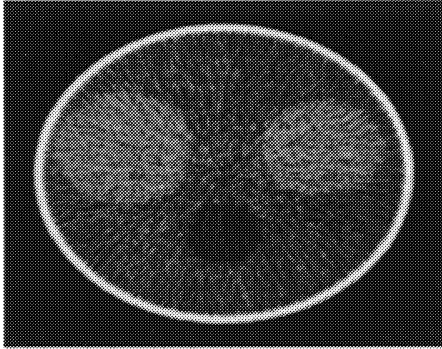

(b)

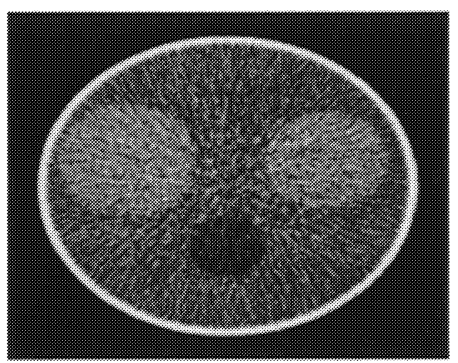

(d)

Figure 2. Top row: Images reconstructed using the EM algorithm with 40 iterations from the noisy (a) full-time and (b) half-time data functions. Bottom row: Images reconstructed using the EM algorithm with 60 iterations from the noisy (c) full-time and (d) half-time data functions. The matrix size of the reconstructed images is $256 \times 256$ pixels.

\section{Experimental TAT measurement data}

We also utilized experimental TAT measurement data that were acquired previously in the laboratory of Professor Lihong Wang. ${ }^{13}$ The data were acquired using the measurement geometry shown in Fig. 1-(b) and had a scanning radius $R_{0}=70 \mathrm{~mm}$. Measurements were taken at 160 equally spaced positions on the scanning 
aperture and for each measurement the received pressure signal was sampled at 2000 points at a sampling frequency of $50 \mathrm{MHz}$. A low-pass filter was applied to (temporally) smooth the measurement data at each receiver location in order to remove white noise. Full- and half-time data reflectivity tomography data functions were calculated from the filtered pressure signals by use of Eqn. (8).

\section{Reconstruction procedure}

Because the TAT data functions were converted to reflectivity tomography data functions via. Eqn. (8), reconstruction of $A(\vec{r})$ could be accomplished by use of a reflectivity tomography reconstruction algorithm. Analytic inversion formulae for reconstructing images from half-time data functions have not been identified. However, because Eqn. (8) is a positive integral equation, the expectation maximization (EM) algorithm ${ }^{8}$ can be employed for reconstruction of $A(\vec{r})$. Because it is applicable to both full- and half-time data functions, the EM algorithm was used to reconstruct images from the simulated and experimental (full- and half-time) data functions.

\subsection{Reconstructed images}

Figure 2 contains the images that were reconstructed from the noisy simulation data. Figures 2-(a) and 2(b) were reconstructed from the full- and half-time data functions, respectively, using 40 iterations of the EM algorithm. Figures 2-(c) and 2-(d) were reconstructed from the full- and half-time data functions, respectively, using 60 iterations of the EM algorithm. It is clearly evident that the images reconstructed from the halftime data functions contain reduced noise levels compared to the corresponding images reconstructed from the full-time data functions. This observation is consistent with our conjecture that, in certain cases, half-time reconstruction approaches for TAT may have better statistical properties than conventional (full-time-based) approaches.

Figure 3 contains the images that were obtained from the experimental TAT data by use of the (a) fulltime and (b) half-time reconstruction approaches. When reconstructing the images, the EM algorithm was terminated after 100 iterations. Because the TAT pressure data were preprocessed by use of low-pass filter, neither of these images have a very noisy appearance. However, the image reconstructed from the half-time data function in Fig. 3-(b) has significantly better spatial resolution than the image reconstructed from the full-time data function in Fig. 3-(a).

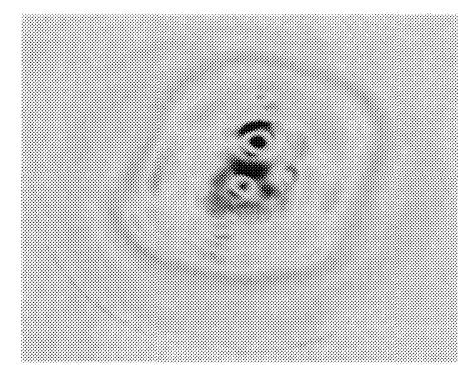

(a)

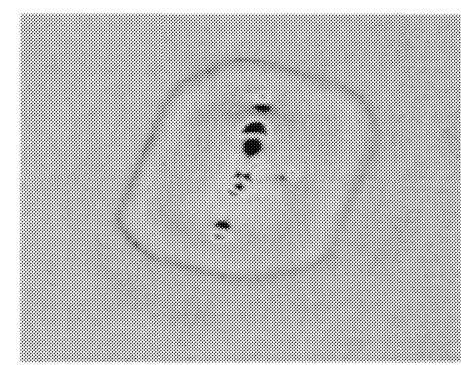

(b)

Figure 3. Images reconstructed using the EM algorithm with 100 iterations from the experimental (a) full-time and (b) half-time data functions. The matrix size of the reconstructed images is $400 \times 400$ pixels.

\section{SUMMARY}

We have proposed and investigated a reconstruction approach for TAT that employs a half-time reconstruction algorithm from reflectivity tomography. Using realistic assumptions regarding the statistical properties of TAT measurement data, we demonstrated that the half-time reconstruction algorithm could produce images that contained reduced noise levels compared to those in images produced by use of a full-time reconstruction 
algorithm. If the TAT data function is smoothed explicitly to remove certain components of the data noise, the use of a half-time reconstruction algorithm can improve the spatial resolution of the reconstructed image. These observations suggest that half-time reconstruction algorithms for reflectivity tomography may be of significant value for reducing image noise and improving the detectability of low-contrast objects in TAT imaging applications.

\section{REFERENCES}

1. T. Mast, "Wideband quantitative ultrasonic imaging by time-domain diffraction tomography," Journal of the Acoustical Society of America 106, pp. 3061-3071, 1999.

2. J. Ylitalo, J. Kaovukangas, and J. Oksman, "Ultrasonic reflection mode computed tomography through a skullbone," IEEE Transactions on Biomedical Engineering 37, pp. 1059-1065, 1990.

3. K. Dines and A. Goss, "Computer ultrasonic reflection tomography," IEEE Transactions on Ultrasonics, Ferroelectrics and Frequency Control 34, pp. 309-317, 1987.

4. M. Moshfeghhi, "Ultrasound reflection-mode tomography using fan-shaped-beam insonification," IEEE Transactions on Ultrasonics, Ferroelectrics and Frequency Control 33, pp. 299-314, 1986.

5. S. Norton, "Reconstruction of a two-dimensional reflecting medium over a circular domain: Exact solution," Journal of the Acoustical Society of America 67(4), pp. 1266-1273, 1980.

6. S. Norton and M. Linzer, "Ultrasonic reflectivity imaging in three dimensions: Exact inverse scattering solutions for plane, cylindrical, and spherical apertures," IEEE Transactions on Biomedical Engineering 28, pp. 202-220, 1981.

7. D. Finch, S. Patch, and Rakesh, "Determining a function from its mean values over a family of spheres," SIAM Journal of Mathematical Analysis 35, pp. 1213-1240, 2004.

8. X. Pan, Y. Zou, and M. Anastasio, "Data redundany and reduced-scan reconstruction in reflectivity tomography," IEEE Transactions on Image Processing 12, pp. 784-795, 2003.

9. X.Pan, Y. Zou, M. Anastasio, and E. Sidky, "Data symmetries and reduced-scan image reconstruction in 3D reflectivity tomography," Physics in Medicine and Biology (submitted) , 2004.

10. M. Anastasio, Y. Zou, and X. Pan, "Reflectivity tomography using temporally truncated data," in $I E E E$ EMBS/BMES Conference Proceedings, vol. 2, pp. 921-922, IEEE, 2002.

11. Y. Zou, X. Pan, and M. Anastasio, "Data truncation and the exterior reconstruction problem in reflectionmode tomography," in IEEE Nuclear Science Symposium Conference Record, vol. 2, pp. 726-730, IEEE, 2002.

12. E. Y. Sidky and X. Pan, "Image reconstruction with a half-detector in single-photon emission computed tomography with non-uniform attenuation," Optical Engineering 42, pp. 2506-2513, 2003.

13. M. Xu and L. Wang, "Time-domain reconstruction for thermoacoustic tomography in a spherical geometry," IEEE Transactions on Medical Imaging 21, pp. 814-822, 2002.

14. W. Joines, R. Jirtle, M. Rafal, and D. Schaeffer, "Microwave power absorption differences between normal and malignant tissue," Radiation Oncology, Biology, Physics 6, pp. 681-687, 1980.

15. W. Cheong, S. Prahl, and A. Welch, "A review of the optical properties of biological tissues," IEEE J. Quantum Elect. 26, pp. 2166-2185, 1990.

16. $\mathrm{M}$. $\mathrm{Xu}, \mathrm{Y} . \mathrm{Xu}$, and $\mathrm{L}$. Wang, "Time-domain reconstruction algorithms and numerical simulations for thermoacoustic tomography in various geometries," IEEE Transactions on Biomedical Engineering 50, pp. 1086-1099, 2003.

17. R. Kruger, P. Liu, R. Fang, and C. Appledorn, "Photoacoustic ultrasound (PAUS) reconstruction tomography," Medical Physics 22, pp. 1605-1609, 1995. 